\title{
Initial experience in Brazil with endoscopic submucosal dissection for early gastric cancer using insulation-tipped knife: a safety and feasibility study
}

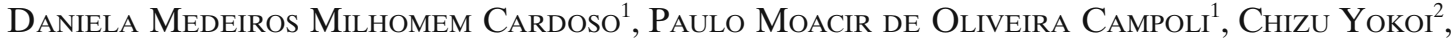 \\ Flávio Hayato Ejima ${ }^{1}$, Paulo Adriano de Queiroz Barreto ${ }^{3}$, Alexandre Menezes de Brito ${ }^{3}$, \\ Eliane Duarte Mota ${ }^{4}$, Ailton Cabral de Fraga Junior ${ }^{4}$, and Orlando Milhomem da Mota ${ }^{3}$ \\ ${ }^{1}$ Endoscopic Unit, Department of Gastrointestinal Oncology, Araújo Jorge Cancer Hospital, Rua 239, No. 181, Setor Universitário, \\ CEP 74605-070, Goiânia, GO, Brazil \\ ${ }^{2}$ Endoscopy Unit, National Cancer Center Hospital, Tokyo, Japan \\ ${ }^{3}$ Department of Gastrointestinal Oncology, Araújo Jorge Cancer Hospital, Goiânia, GO, Brazil \\ ${ }^{4}$ Department of Pathology, Araújo Jorge Cancer Hospital, Goiânia, GO, Brazil
}

\begin{abstract}
Background. Endoscopic resection is an adequate treatment for subgroups of patients with early gastric cancer. Endoscopic submucosal dissection (ESD) represents a recent advance and leads to good results when adequately indicated. There is great experience with this technique in Japan and it is gaining acceptance among Western endoscopists. We present the first gastric ESD series performed in Brazil.

Methods. Patients with well-differentiated adenocarcinomas macroscopically classified as early cancer, less than $30 \mathrm{~mm}$ with no ulcer or scar, were included. ESD was performed with an insulated-tip knife and electrosurgical unit with endocut mode. Clinicopathological aspects and morbidity were evaluated. The study was approved by the local ethics committee and informed consent was obtained from all participant subjects.

Results. From October 2005 to July 2007, 160 patients received surgical treatment for gastric cancer; 44 patients $(27.5 \%)$ had early gastric cancer. In this latter group, 15 procedures (ESD) were performed in 12 patients. The mean size of the lesions was $16.8 \mathrm{~mm}$. Almost half of the lesions were located in the proximal third of the stomach and the mean elapsed time for the procedure was $140 \mathrm{~min}$. In $80 \%$ of the cases resection was en bloc and $80 \%$ of the resections were considered curative. We had three perforations, which were managed clinically, and no bleeding.

Conclusion. When adequately indicated, ESD is a safe and feasible technique.
\end{abstract}

Key words Endoscopic resection · Early gastric cancer · Endoscopic submucosal dissection (ESD)

Offprint requests to: D.M.M. Cardoso

Received: May 17, 2008 / Accepted: October 10, 2008

\section{Introduction}

Early gastric cancer (EGC), according to the Japanese classification of gastric carcinoma established by the Japanese Gastric Cancer Association [1], is defined as a lesion confined to the mucosa or submucosa regardless of the presence or absence of regional lymph node metastasis. The presence of lymph node metastasis has an important adverse effect on the patient's prognosis, so a gastrectomy with lymph node dissection was the only option for the treatment of EGC for many years.

Recently some studies with long-term data from large series of patients have shown that there are specific groups of patients with EGC with practically no risk of lymph node metastasis. Gotoda and colleagues [2] established endoscopic and pathological criteria for these groups of patients that are candidates for endoscopic treatment.

These advances in the theoretical field, in addition to technical improvements in endoscopic equipment and skills during recent years have also allowed therapeutic endoscopy to play a major role in the treatment of EGC.

Endoscopic mucosal resection (EMR) by a strip biopsy technique was first described in Japan by Tada et al. [3] in 1984 and was an extension of the endoscopic snare technique. Other technical options such as EMR with hypertonic saline epinephrine solution (ERSHE), described by Hirao et al. [4] in 1988; EMR with a capfitted endoscope (EMR-C), described by Inoue et al. [5] in 1993; and EMR using a ligation device, described in Brazil by Chaves et al. [6] in 1994 were developed.

EMR is an efficacious, safe, and convenient technique, but it has some limitations regarding the assessment of the depth of invasion and the management of 
large lesions where piecemeal resections are common, carrying high recurrence rates $[7,8]$.

In 1998 Hosokawa and Yoshida [9] developed a special endoscopic knife with a ceramic ball on top called the insulation-tipped diathermic knife (IT-knife). Endoscopic resections with direct dissection of the submucosal layer using modified needle knifes were called endoscopic submucosal dissections (ESDs).

ESD with the IT-knife was the first of these techniques and represents a recent advance in the management of EGC [8]. It is frequently performed in many institutions, especially in Japan.

During ESD the endoscopist dissects directly along the submucosal layer. The endoscopic resection is not limited by lesion size and a piecemeal resection is avoided. This procedure is gaining acceptance among Western endoscopists and it has great potential to replace surgery for the treatment of some stages of EGC, allowing a complete cure of the patient with no need for additional treatment and preserving gastric function with a high quality of life. On the other hand, ESD involves a higher complication rate and longer operation time, and it requires refined skills $[7,8,10,11]$.

Since 2002 we have performed endoscopic resection for the treatment of EGC in our institution [12]. During the first years we used the technique proposed by Tada et al. [3] and the technical variant proposed by Hirao et al. [4] (ERHSE) with circumferential incision. In October 2005 we started our experience using ESD with the IT-knife. The aim of the present study was to assess the indications, pathological results, and morbidity in a series of patients that underwent ESD with the IT-knife and to evaluate the safety and feasibility of the method.

\section{Patients, materials, and methods}

This study included patients from the Department of Gastrointestinal Oncology of this institution (Araújo Jorge Cancer Hospital, Goiânia, GO, Brazil). All the patients presented with clinical and endoscopic features of EGC and were referred to the Digestive Endoscopy Division to assess the indication for the endoscopic resection.

The patients included were those with EGC macroscopically recognized by endoscopy and who met the endoscopic and pathological criteria for endoscopic resection. The tumors were classifiable as EGC according to the Japanese classification of gastric carcinoma established by the Japanese Gastric Cancer Association [1].

Only patients who had well-differentiated adenocarcinomas without ulceration or scar and with a diameter of up to $30 \mathrm{~mm}$ were included. Cases macroscopically classified as advanced cancer were excluded.

ESD was performed in the endoscopy room with the patient under conscious sedation; the patients were monitored through pulse oximetry. Supplementary oxygen was used when necessary. All procedures were performed with a single-channel endoscope (GIF V and GIF V2; Olympus America, Melville, NY, USA) and an Olympus CV-100 video processor was used. Endoscopic ultrasonography was not used for staging prior to the resection in the current series.

Chromoscopy was performed in all patients, using $0.2 \%$ indigo carmine solution, to obtain an accurate definition of the lesion dimension, macroscopic appearance, and margins; and an electrosurgical generator with endocut mode was used (ICC-200; ERBE, Tubingen, Germany).

The ESD steps [Fig. 1A-F] consisted of:

- Marking the limits of the lesion using a needle-knife (KD-1L-1; Olympus, Tokyo, Japan) in a distance of approximately $0.5 \mathrm{~mm}$ from the lesion [Fig. 1A];

- Injection of fluid (hypertonic saline solution with epinephrine) in the submucosal layer around the lesion in order to separate it from the muscle layer [Fig. 1B];

- Cutting the mucosa surrounding the tumor to obtain a circumferential incision with submucosal exposure, using both the needle-knife and the IT-knife (KD610L, Olympus) [Fig. 1C, D];

- Submucosal dissection of the connective tissue of the submucosal layer. Maintenance of the submucosal cushion with additional fluid injection is an important tool for the security of the process [Fig. 1E, F].

Control of the bleeding was obtained with either the needle-knife, the IT-knife, or by using Hot Biopsy Forceps (U6122; G-Flex, Atlanta, GA, USA).

The use of a transparent attachment (D-206-04; Olympus) to create countertraction is sometimes important during the procedure.

All the resected specimens were stretched and fixed in $10 \%$ formalin in a plate and they were sectioned at intervals of $2 \mathrm{~mm}$.

Clinical aspects were evaluated. These included the patient's age, sex, location of the tumor in the stomach, and the macroscopic appearance. Pathological aspects such as the tumor size and macroscopic type, the histological type, depth of tumor invasion, lateral and vertical margins, and vascular and lymphatic involvement were also evaluated. All histological sections were reviewed by one single pathologist (E. D. M.).

Outcomes such as completeness of the resection and complications such as hemorrhage and perforation were analyzed. 


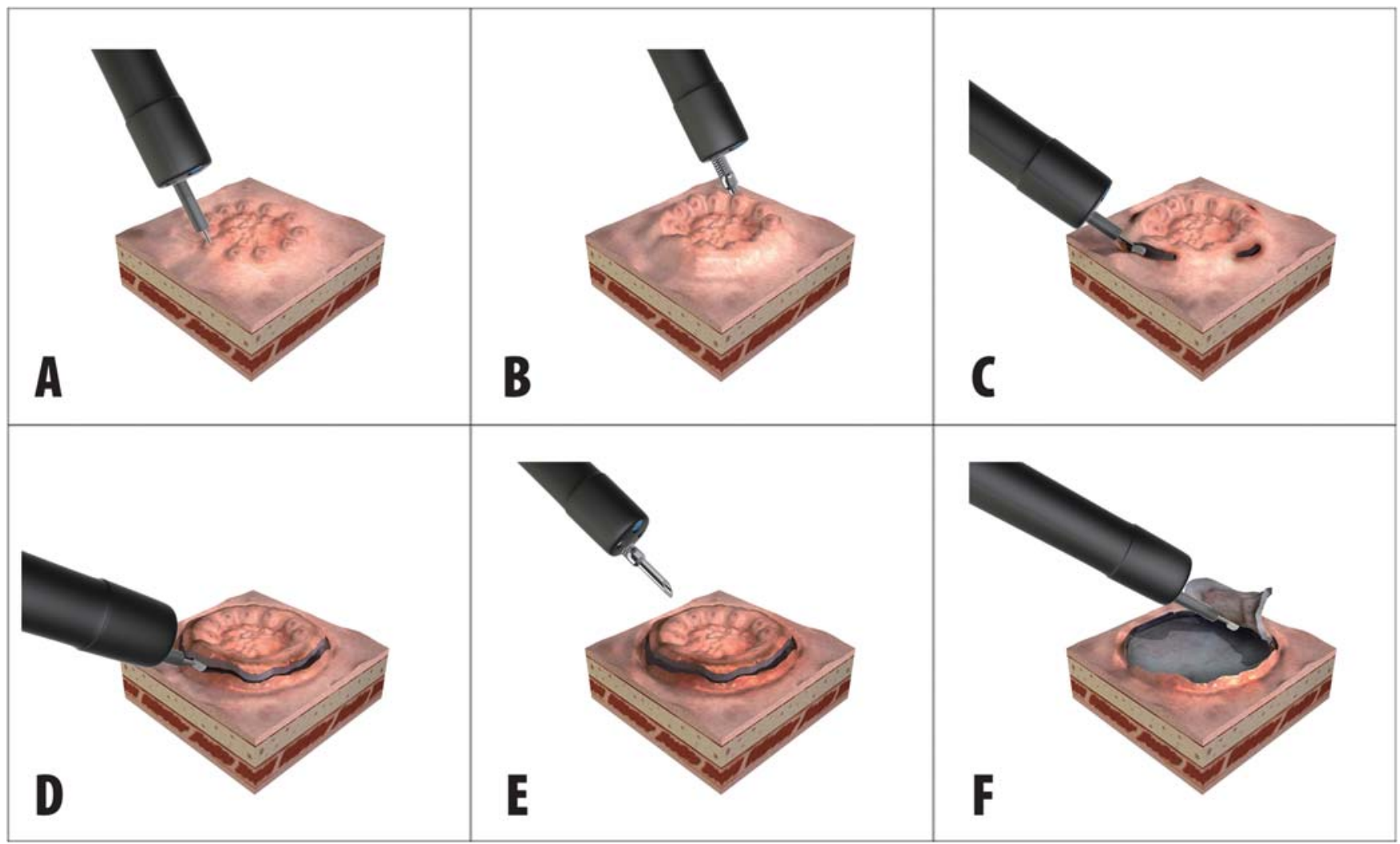

Fig. 1A-F. Steps for endoscopic submucosal dissection (ESD). A Marking the limits of the lesion; $\mathbf{B}$ injection of fluid; $\mathbf{C}$ and $\mathbf{D}$ cutting the surrounding mucosa; $\mathbf{E}$ additional fluid injection; $\mathbf{F}$ dissection of the submucosal layer

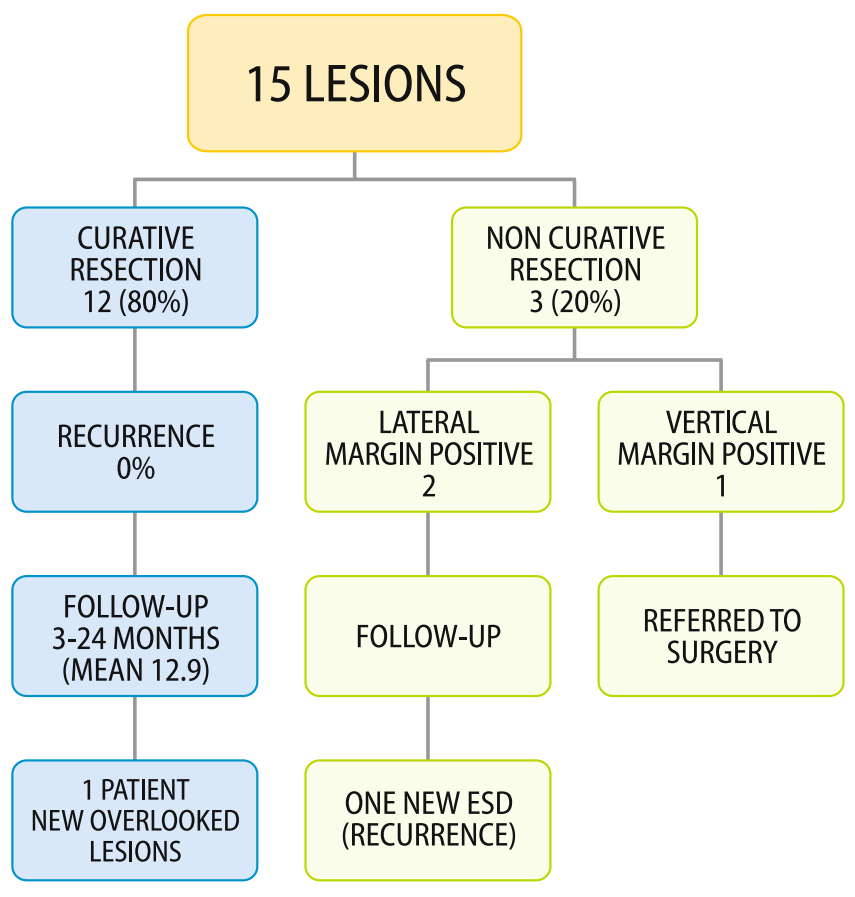

Fig. 2. Short-term outcomes of the patients
Resections were considered noncurative when the margins were compromised by the tumor or could not be evaluated.

This study was approved by the ethics committee of our institution and written informed consent was obtained from all patients.

\section{Results}

From October 2005 to July 2007, 160 patients received surgical treatment for gastric cancer in our institution; 44 patients $(27.5 \%)$ had EGC. In this latter group, 12 patients met the criteria for endoscopic resection by ESD and 15 procedures were performed in these 12 patients.

The ESD group consisted of six men and six women, with ages ranging from 27 to 91 years (mean, 71.2 years). The characteristics of the resected specimens are listed in Table 1. Macroscopically there were 10 IIa lesions $(67 \%)$ and $3 \mathrm{IIa}+$ IIc lesions $(20 \%)$. The size of the lesions varied from $8 \mathrm{~mm}$ to $20 \mathrm{~mm}$ (mean, $16.8 \mathrm{~mm}$ ) and the most common site of the lesions was the proximal third of the stomach, with 7 lesions (46.6\%), 
Table 1. Characteristics of the resected specimens

\begin{tabular}{|c|c|c|c|c|c|c|}
\hline Location & Size $(\mathrm{mm})$ & $\begin{array}{c}\text { Macroscopic } \\
\text { type }\end{array}$ & Depth & $\begin{array}{l}\text { One-piece } \\
\text { resection }\end{array}$ & $\begin{array}{l}\text { Vertical } \\
\text { margin }\end{array}$ & $\begin{array}{l}\text { Lateral } \\
\text { margin }\end{array}$ \\
\hline \multirow[t]{7}{*}{ Proximal third } & 20 & $\mathrm{IIa}$ & M & $\mathrm{No}^{\mathrm{a}}$ & Negative & Negative \\
\hline & 15 & $\mathrm{IIa}+\mathrm{II} c$ & M & Yes & Negative & Negative \\
\hline & 15 & IIc & M & Yes & Negative & Negative \\
\hline & 15 & $\mathrm{IIa}+\mathrm{IIc}$ & M & Yes & Negative & Positive \\
\hline & 20 & IIa & M & Yes & Negative & Negative \\
\hline & 15 & IIa & $\mathrm{M}$ & $\mathrm{No}^{\mathrm{b}}$ & Negative & Negative \\
\hline & 15 & IIa & SM & Yes & Positive & Negative \\
\hline \multirow[t]{4}{*}{ Medial third } & 15 & $\mathrm{IIa}+\mathrm{IIc}$ & $\mathrm{M}$ & Yes & Negative & Negative \\
\hline & 15 & IIa & M & Yes & Negative & Negative \\
\hline & 8 & IIc & M & Yes & Negative & Negative \\
\hline & 20 & IIa & M & Yes & Negative & Negative \\
\hline \multirow[t]{4}{*}{ Distal third } & 20 & IIa & M & Yes & Negative & Negative \\
\hline & 20 & IIa & M & $\mathrm{No}^{\mathrm{b}}$ & Negative & Positive \\
\hline & 20 & IIa & M & Yes & Negative & Negative \\
\hline & 20 & IIa & M & Yes & Negative & Negative \\
\hline
\end{tabular}

${ }^{\mathrm{a}}$ Two fragments

${ }^{\mathrm{b}}$ Three fragments

followed by the medial third and the distal third, with 4 lesions each (Table 1). All the tumors were welldifferentiated adenocarcinomas. The median elapsed time for the procedure was $140 \mathrm{~min}$.

One-piece (en-bloc) resection was obtained in 12 patients $(80 \%)$. In 12 patients the resections were considered curative (80\%). The noncurative resections included two patients with positive lateral margins and one with a positive vertical margin and submucosal invasion. Of the 15 resected specimens, 14 were limited to the mucosal layer (Table 1). Vascular and lymphatic invasion was not observed in the current series.

Of the three patients with noncurative resection, one was referred to the surgical team for gastrectomy (the one with submucosal invasion) and the other two were followed. One of the followed patients developed a recurrent lesion 10 months after the first ESD, and the lesion was resected with a new ESD. The other patient is in the twenty-fourth month of follow up without any other changes (Fig. 2).

During the third month of the follow-up period one of the patients with prior curative resection had a diagnosis of two other overlooked early lesions, one of them located in the gastric body and the other one located in the gastric antrum. The histopathological study revealed an undifferentiated-type adenocarcinoma in one of the new lesions, and this patient was referred for surgical treatment.

Morbidity related to the procedure consisted of three cases of gastric perforation, two of them highlighted by the formation of a pneumoperitoneum diagnosed by $\mathrm{X}$-ray, without clinical symptoms and one of them diagnosed during the procedure. The first two patients were treated clinically by fasting, placement of a nasogastric tube, and IV antibiotic therapy, with oral feeding on postoperative day 3 , with a good outcome. The perforation case diagnosed during the procedure was closed with endoclips (HX-201LR-135; Olympus) and this patient also received clinical treatment with a good outcome. In two of the patients with perforation the lesions were located in the proximal third and in the other the lesion was located in the medial third. There was no massive bleeding during the procedures and there were no cases of delayed bleeding in the current series.

\section{Discussion}

ESD reflects the new advances in therapeutic endoscopy for the management of EGC. It consists of a minimally invasive procedure which provides adequate pathological staging and has high curative rates, preserving the gastric function and the quality of life of the patients. ESD can also be an important tool to treat recurrent disease after incomplete endoscopic resections $[13,14]$. Besides, ESD does not preclude the possibility of a future surgical treatment.

These minimally invasive procedures sometimes increase morbidity, and are more demanding and timeconsuming than open procedures, so they must have clear indications. One of the most important factors in choosing ESD and other endoscopic resection techniques for EGC is the precise prediction of whether or not the patient has lymph node metastasis. The negligible incidence of lymph node metastasis in selected subgroups of patients with EGC raises the possibility of local treatment with results comparable to the surgical ones [2]. 
Incidence rates of gastric cancer in Japan are among the highest in the world (61.8 per 100000 men and 23.8 per 100000 women) and more than $60 \%$ of the cases are early tumors [15]. In Brazil the incidences are lower than in Japan, with fewer than $15 \%$ of the cases classified as early type [16-19]. The population-based Cancer Registry of Goiania refers to an incidence of 19.0 per 100000 men and 9.0 per 100000 women [20].

In Brazil, the small number of early cancer cases and the lack of technical resources compared to Japanese specialized centers produces difficulties in achieving proficiency in endoscopic resections. The present series shows the initial experience with ESD at a tertiary referral oncology hospital in Brazil.

In the present study all the patients met the extended indication criteria proposed in 2000 by Gotoda and colleagues [2] to achieve the best results with endoscopic treatment. The current series showed a curative resection rate of $80 \%$ and an en-bloc resection rate of $80 \%$, comparable with the results in other series from the Japanese and Western literature [7, 8, 10, 11, 21] (Table 2).

Since 2002 we have had experience with endoscopic resection for the treatment of EGC in our institution, and between 2002 and 2005 thirteen EMRs were performed. The first seven cases were resected using the strip biopsy technique and the latter six cases were resected using a technical variant of ERSHE (hypertonic saline epinephrine solution) with circumferential incision. Both the en-bloc and curative resection rates were $69 \%$ [12]. There were two patients with perforations who received conservative treatment with good outcomes. Our experience with ESD started in 2005 and it seems to be more suitable for the management of EGC. ESD is not limited by the size of the lesion and we can obtain higher en-bloc and curative resection rates, avoiding recurrences. However, comparative studies of the two techniques in our population are necessary.

The concerns with regard to initial experiences and the learning curve fall into two categories: risk of com- plications (bleeding and perforation) and the risk of inadequate treatment. The complications of ESD are usually treated in a conservative fashion with endoscopic procedures and clinical measures. The major concern should be to provide the patient with adequate treatment with complete resection of the tumor [22].

Bleeding is a relatively common complication after ESD. Most bleeding occurs within $12 \mathrm{~h}$ after the procedure and is strongly related to the size and location of the tumor [23]. In the current series we had no procedure-related gastric bleeding.

Large series of ESD report perforation rates lower than $6 \%$, related to tumor location and ulcer findings $[7,8,10,11,21,24]$. In the current series we had three cases of perforation, which represents $20 \%$. Some observations should be taken into account, such as the report of Choi et al. [25], which had a $15 \%$ perforation rate during the first 20 cases of EMR and then the rate dropped to $1.7 \%$ in the next 60 cases. High perforation rates during the first experiences with the method were also shown by other authors [26].

The high perforation rates presented in the first cases in the current series demonstrate that there is a learning curve to be covered. Some authors state that a trainee requires at least 30 cases to gain proficiency in ESD and to decrease the number of complications [22,27].

Gastric perforation during ESD does not seem to increase the risk of peritoneal dissemination, this being shown by Ikehara and colleagues [28], who reviewed 90 cases of perforation during endoscopic resections with long-term follow-up. Table 2 shows bleeding, perforation, and curative resection rates from large ESD series.

Endoscopic closure and clinical treatment for gastric perforation that occurs during ESD usually have good outcomes [28]. The three patients with perforation in the current series were treated in a conservative fashion with good outcomes.

Longer procedure time can also be improved by covering the learning curve. The elapsed time for the pro-

Table 2. Bleeding, perforation, and curative resection rates from large ESD series

\begin{tabular}{|c|c|c|c|c|c|c|}
\hline & Year & $n$ & Bleeding (\%) & Perforation (\%) & Complete resection (\%) & Curative resection (\%) \\
\hline Ohkuwa [21] & 2001 & 41 & 22 & 5 & - & - \\
\hline Rosch [24] & 2004 & 13 & 15.4 & 0 & 30.8 & - \\
\hline Miyazaki [26] & 2005 & 29 & 11.5 & 11.5 & 93 & - \\
\hline Choi [25] & 2005 & 20 & 5 & 15 & 65 & - \\
\hline Oda $[8]$ & 2006 & 303 & 0 & 3.6 & 73.6 & 73.6 \\
\hline Watanabe [7] & 2006 & 120 & 0 & 4.1 & 87.5 & - \\
\hline Imagawa [10] & 2006 & 196 & 0 & 6.1 & 84 & 84 \\
\hline Onozato [11] & 2006 & 251 & 9.2 & 3.6 & 90 & 92 \\
\hline Neuhaus [31] & 2006 & 10 & 40 & 50 & 60 & - \\
\hline Kakushima [27] & 2006 & 49 & 7 & 2 & 96 & - \\
\hline Current series & 2008 & 15 & 0 & 20 & 80 & 80 \\
\hline
\end{tabular}


cedure in the current series was $140 \mathrm{~min}$ and this reflects the technical difficulties during the initial experience.

One important point needs to be made in regard to the use of the electrosurgical unit with endocut mode (ICC 200; ERBE, Tubingen, Germany), which provides a very sharp cut of the mucosal and submucosal layers, without coagulation artifacts, in the resected specimen. The use of this unit makes the procedure easier and safer and increases the quality of the pathological evaluation of the resected specimen.

Some centers indicate endoscopic ultrasonography (EUS) as a routine before performing endoscopic resections, in order to assess the depth of invasion and obtain a precise staging. EUS is a useful tool, but it offers limited accuracy, of $78 \%-80 \%$ for tumor staging, which means that $20 \%-22 \%$ of the patients may receive inadequate evaluation prior to the resection $[29,30]$. EUS was not used for staging in the current series.

New technologies have been developed to improve the safety and feasibility of ESD [31,32] and probably in the future ESD will be a standard technique to treat EGC in Western countries, as it is in Japan. In Brazil few centers are likely to diagnose enough cases of EGC to become expert in ESD. The diagnosis of early cases is not easy and usually involves not only the endoscopy team. It is necessary to create an "early diagnosis culture" involving the family doctor, the clinical and surgical gastroenterologist, the endoscopist, and the pathologist, so that we can reach a good level in diagnosis and treatment expertise. The present study represents the first ESD case series performed in our country. It will be necessary to make efforts in order to improve early diagnosis, endoscopy resources, experience, and skills so that ESD techniques can be disseminated to cure many patients with EGC safely and effectively.

\section{Conclusions}

In conclusion, ESD is a feasible and safe endoscopic technique for the treatment of EGC when adequately indicated. It allows adequate pathological staging, is minimally invasive, and preserves the gastric function with a high quality of life. On the other hand, it carries a high risk of complications and is time-consuming as well as requiring refined skills.

The current series demonstrated the initial experience, the difficulties, and early outcomes with the ESD method in a tertiary cancer hospital in Brazil. Careful follow up of the patients is also required to investigate the long-term results regarding overall survival and recurrence.
Acknowledgments The authors thank Dr. Takuji Gotoda and Dr. Ichiro Oda from the National Cancer Center, Tokyo, Japan, for their helpful advice. The authors also thank Mr. Henrique Toledo for the help with figure and graphics management.

\section{References}

1. Japanese Gastric Cancer Association. Japanese classification of gastric carcinoma - 2nd English edition - Gastric Cancer 1998;1:10-24.

2. Gotoda T, Yanagisawa A, Sasako M, Ono H, Nakanishi Y, Shimoda $\mathrm{T}$, et al. Incidence of lymph node metastasis from early gastric cancer: estimation with a large number of cases at two large centers. Gastric Cancer 2000;3:219-25.

3. Tada M, Murata M, Murakami F, Shimada M, Mizumachi S, Arima K, et al. Development of strip-off biopsy. Gastroenterol Endosc 1984;26:833-9.

4. Hirao M, Masuda K, Asanuma T, Naka H, Noda K, Matsuura K, et al. Endoscopic resection of early gastric cancer and other tumors with local injection of hypertonic saline-epinephrine. Gastrointest Endosc 1998;34:264-9.

5. Inoue H, Takeshita K, Hori H, Muraoka Y, Yoneshima H, Endo M. Endoscopic mucosal resection with a cap-fitted panendoscope for esophagus, stomach and colon mucosal lesions. Gastrointest Endosc 1993;39:58-62.

6. Chaves DM, Sakai P, Mester M, Spinosa SR, Tomishigue T, Ishioka S. A new endoscopic technique for the resection of flat polypoid lesions. Gastrointest Endosc 1994;40:224-6.

7. Watanabe K, Ogata S, Kawazoe S, Watanabe K, Koyama T, Kajiwara T, et al. Clinical outcomes of EMR for gastric tumors: historical pilot evaluation between endoscopic submucosal dissection and conventional mucosal resection. Gastrointest Endosc 2006;63:776-82.

8. Oda I, Saito D, Tada M, Iishi H, Tanabe S, Oyama T, et al. A multicenter retrospective study of endoscopic resection for early gastric cancer. Gastric Cancer 2006;9:262-70.

9. Hosokawa K, Yoshida S. Recent advances in endoscopic mucosal resection for early gastric cancer. Jpn J Cancer Chemother 1998; 25:476-83.

10. Imagawa A, Okada H, Kawahara Y, Takenaka R, Kato J, Kawamoto H, et al. Endoscopic submucosal dissection for early gastric cancer: results and degrees of technical difficulty as well as success. Endoscopy 2006;38:987-90.

11. Onozato Y, Ishihara H, Iizuka H, Sohara N, Kakizaki S, Okamura $\mathrm{S}$, et al. Endoscopic submucosal dissection for early gastric cancers and large flat adenomas. Endoscopy 2006;38:980-6.

12. Campoli PM, Ejima FH, Cardoso DM, Mota ED, Fraga AC Jr, da Mota OM. Endoscopic mucosal resection of early gastric cancer: initial experience with two technical variants. Arq Gastroenterol 2007;44:250-6.

13. Yokoi C, Gotoda T, Hamanaka H, Oda I. Endoscopic submucosal dissection allows curative resection of locally recurrent early gastric cancer after prior endoscopic mucosal resection. Gastrointest Endosc 2006;64:212-8.

14. Fujishiro M, Goto O, Kakushima N, Kodashima S, Muraki Y, Omata M. Endoscopic submucosal dissection of stomach neoplasms after unsuccessful endoscopic resection. Dig Liver Dis 2007;39:566-71.

15. Ajiki W, Tsukuma H, Oshima A. Research Group for PopulationBased Cancer Registration in Japan. Cancer incidence and incidence rates in Japan in 1999: estimates based on data from 11 population-based cancer registries. Jpn J Clin Oncol 2004;34: 352-6.

16. Henry MACA, Saad LHC, Goncalves Junior I, Bozoni LLM. Gastric cancer: the analysis of the results of the surgical treatment (in Portuguese). ABCD Arq Bras Cir Dig 1991;6:82-5. 
17. Jacob CE. Early gastric cancer: clinical and pathologic features; correlation with late survival (doctoral dissertation; in Portuguese). Sao Paulo: Universidade de Sao Paulo, Faculdade de Medicina, Departamento de Gastroenterologia; 2003.

18. Marchesini JB, Brenner S, Buffara Junior VA, Moreira M. Early gastric cancer: analysis of 12 cases (in Portuguese). Rev Bras Cir 1992;82:77-81.

19. Modena JLP. Early gastric cancer. In: Magalhaes AF, Cordeiro FT, Quilici FA, Machado G, Amarante HMBS, Prolla JC, Leitao OR, Alves PRA, Sakai P, editors. Endoscopia Digestiva - Diagnostica e Terapeutica (Digstive endoscopy diagnostic and therapeutic). Rio de Janeiro: Ed Revinter; 2005. p. 338-55.

20. Curado MP, Silva CMB, Martins E, Sousa EC, Mineiro MS, Oliveira JC. Cancer incidence in Brazil, Goiania (1999-2002). In: Curado MP, Edwards B, Shin HR, Storm H, Ferlay J, Heanue M, Boyle P, editors. Cancer incidence in five continents: vol. IX. Lyon: IARC Scientific Publications; 2007. no. 160.

21. Ohkuwa M, Hosokawa K, Boku N, Ohtu A, Tajiri H, Yoshida S. New endoscopic treatment for intramucosal gastric tumors using an insulated-tip diathermic knife. Endoscopy 2001;33: 221-6.

22. Gotoda T, Friedland S, Hamanaka H, Soetikno R. A learning curve for advanced endoscopic resection. Gastrointest Endosc 2005;62:866-7.

23. Shiba M, Higuchi K, Kadouchi K, Montani A, Yamamori K, Okazaki $\mathrm{H}$, et al . Risk factors for bleeding after endoscopic mucosal resection. World J Gastroenterol 2005;11:7335-9.

24. Rosch T, Sarbia M, Schumacher B, Deinert K, Frimberger E, Toermer $\mathrm{T}$, et al. Attempted endoscopic en bloc resection of mucosal and submucosal tumors using insulated-tip knives: a pilot series. Endoscopy 2004;36:788-801.
25. Choi IJ, Kim CG, Chang HJ, Kim SG, Kook MC, Bae JM. The learning curve for EMR with circumferential mucosal incision in treating intramucosal gastric neoplasm. Gastrointest Endosc 2005;62:860-5.

26. Miyazaki S, Gunji Y, Aoki T, Nakajima K, Nabeya Y, Hayashi H, et al. High en bloc resection rate achieved by endoscopic mucosal resection with IT knife for early gastric cancer. Hepatogastroenterology 2005;52:954-8.

27. Kakushima N, Fujishiro M, Kodashima S, Muraki Y, Tateishi A, Omata M. A learning curve for endoscopic submucosal dissection of gastric epithelial neoplasms. Endoscopy 2006;38:991-5.

28. Ikehara H, Gotoda T, Ono H, Oda I, Saito D. Gastric perforation during endoscopic resection for gastric carcinoma and the risk of peritoneal dissemination. Br J Surg 2007;94:992-5.

29. Ganpathi IS, So JB, Ho KY. Endoscopic ultrasonography for gastric cancer: does it influence treatment? Surg Endosc 2006; 20:559-62.

30. Hizawa K, Iwai K, Esaki M, Matsumoto T, Suekane H, Iida M. Is endoscopic ultrasonography indispensable in assessing the appropriateness of endoscopic resection for gastric cancer? Endoscopy 2002;34:973-8.

31. Neuhaus H, Costamagna G, Deviere J, Fockens P, Ponchon T, Rosch T. Endoscopic submucosal dissection (ESD) of early neoplastic gastric lesions using a new double-channel endoscope (the "R-scope"). Endoscopy 2006;38:1016-23.

32. Imaeda H, Iwao Y, Ogata H, Ichikawa H, Mori M, Hosoe N, et al. A new technique for endoscopic submucosal dissection for early gastric cancer using an external grasping forceps. Endoscopy 2006;38:1007-10. 